\title{
Ejercicio y problema dos caras de la misma moneda
}

\section{Exercise and problem two sides of the same coin}

DOI: $10.46814 / \operatorname{lajdv3n3-001}$

Recebimento dos originais: 04/04/2021

Aceitação para publicação: 05/05/2021

Jorge Hernández Márquez

Escuela Normal Superior Pública del Estado de Hidalgo, México

E-mail: jhmpren@yahoo.com.mx

\author{
Octaviano García Robelo \\ Área de Ciencias de la Educación. Universidad Autónoma del Estado de HidalgoMéxico \\ E-mail: droctavianogarcia@gmail.com \\ Brenda Concepción Hernández Mariano \\ Escuela Normal Superio Pública del Estado de Hidalgo, MéxicoFrancisca Lorena Gomez M \\ E-mail: Brenda.hm.96.m@gmail.com \\ Francisca Lorena Gómez Melo \\ Escuela Normal Superior Pública del Estado de Hidalgo, México \\ E-mail: florenamelo@gmail.com
}

\section{RESUMEN}

El presente documento es parte del reporte final de investigación, en él, se aborda el plantear y resolver problemas en la escuela primaria como objeto de estudio. La investigación se desarrolla bajo el enfoque etnográfico; se hace uso de la entrevista,la observación y el análisis documental para el levantamiento de la información de campo; la investigación teórica se recuperó de las bases de datos de REDIE, SCIELO, REDALYC, entre otras; así mismo, se consultaron las Actas Latinoamericanas de Matemática Educativa (ALME) y las actas de la Conferencia Interamericana de Educación Matemática (CIAEM). Los resultados evidencian las confusiones que tienen los profesores sobre el ejercicio y el problema, lo que representa la mecanización en contraposición al desarrollo de habilidades de pensamiento matemático.

Palabras clave: Problema, ejercicio, etnografía y programa de estudio.

\begin{abstract}
This document is part of the final research report, in it, the approach to raise and solve problems in elementary school as an object of study is addressed. The research is developed under the ethnographic approach; interviews, observation and documentary analysis are used to gather field information; the theoretical research was retrieved from REDIE, SCIELO, REDALYC databases, among others; likewise, the Latin American Acts of Educational Mathematics (ALME) and the proceedings of the Inter-American Conference on Mathematics Education (CIAEM) were consulted. The results show the confusions that teachers have about the exercise and the problem, which represents mechanization as opposed to the development of mathematical thinking skills.
\end{abstract}

Key words: Problem, exercise, ethnography and curriculum. 


\section{ANTECEDENTES}

Las matemáticas, en el imaginario popular, han tenido el papel de ser una disciplina difícil de entender y aprobar en la escuela, entre otras razones, porque exige altos niveles de razonamientoabstracto del aprendiz, las formas de enseñanza de los profesores centradas en el reconocimiento social-personal y el temor y el control en la clase con los alumnos; convirtiéndose en una disciplinaalejada de la realidad inmediata de los sujetos.

Sin embargo, las citadas afirmaciones se desvanecen cuando se adentra al estudio de los procesos didácticos que se desarrollan en el aula, en las formas de aprendizaje de los estudiantes definidas por prácticas culturales específicas del contexto social de pertenencia, y los contenidos matemáticos planteados en el currículo formal para la enseñanza en la educación primaria, entre los que destacan la resolución de problemas de naturaleza aritmética y geométrica.

Con referencia a los procesos didácticos, por mucho tiempo se ha considerado a la enseñanzay el aprendizaje como dos fenómenos que se producen simultáneamente y constituyen un solo proceso, es decir, el aprendizaje como una consecuencia directa de la enseñanza. En la actualidad se cuestiona esta simbiosis, se apuesta por la necesidad de comprender al aprendizaje como elemento fundamental de estudio para explicar las condiciones que lo favorecen, cómo se produce y cómo se potencia. Al respecto se han construido diferentes perspectivas: el empleo del ejercicio repetido y la práctica; la matemática como comprensión conceptual y como resolución de problemas, etnomatemáticas, socioepistemología, entre otras.

Así mismo, en el contexto del aprendizaje confluyen explicaciones desde el campo de la psicología, tales como: el conductismo, la psicogenética constructivista, la cognitiva del procesamiento de la información, el sociocultural y el histórico cultural.

Con relación a los conocimientos matemáticos, en los planes y programas de estudio para laeducación primaria se ha transitado por diferentes perspectivas; así, se pasó de una matemática axiomática a una matemática entendida como lenguaje en la cual la resolución de problemas se convierte en el eje central de aprendizaje, dado que a través de ellos, el alumno activa una serie deestrategias y procesos mentales alejándola de los aprendizajes mecánicos e irreflexivos de la matemática axiomática.

\section{PROBLEMA}

¿Cuáles son las características que diferencian los ejercicios de los problemas en la enseñanza de las matemáticas con estudiantes de educación primaria? 


\title{
3 FUNDAMENTACIÓN TEÓRICA
}

Vila y Callejo (2004) hacen un recuento de los estudios que abordan la enseñanza de las matemáticas a partir de la resolución de problemas y reconocen la existencia de tres enfoques por los que ha transitado.

El primero refiere entender el problema como una tarea; lo único que importa es la estructura matemática y el esfuerzo por planificar la sesión de clase reduciéndola a efectuar el análisis de la tarea en la más pura tradición conductista.

El segundo hace hincapié en los destinatarios (alumnos), se ve la distinta dificultad que puedesuponer la tarea en diferentes estudiantes, lo cual implica tener presente los conocimientos previosy las distintas capacidades personales, pero siempre asociado a la idea de aplicación rutinaria de problema - algoritmo previamente visto - corriendo el riesgo de un proceso de mecanización.

El tercero considera al profesor como el sujeto que determina el problema y el proceso de resolución; ello tiene presente el contexto donde se desarrolla el problema y la dimensión afectivadel estudiante. Esto supone que plantear y resolver problemas en la escuela no puede desligarse de los estudiantes, la intencionalidad del profesor que lo selecciona y la situación concreta de la enseñanza y el aprendizaje.

Así el término problema designa:

\begin{abstract}
Una situación planteada con finalidad educativa, que propone una cuestión matemática cuyo método de solución no es inmediatamente accesible al alumno/resolutor o grupo de alumnos que intentan su solución, porque no dispone de un algoritmo que relacione los datos y la incógnita o de un proceso que identifique automáticamente los datos con la conclusión, y por lo tanto deberá buscar, investigar, establecer relaciones, implicar sus efectos, etcétera; para afrontaruna situación nueva (Vila y Callejo, 2004: 31-32).
\end{abstract}

En este sentido, el problema es una herramienta para pensar matemáticamente.

Ávila afirma que la "resolución de problemas se apoya en la idea de que los niños tienen conocimientos adquiridos fuera de la escuela, en el entorno familiar y social que les permiten solucionar diversos problemas" (2004: 20) al mismo tiempo los posibilita en el diseño de procedimientos iníciales informales cuando se le plantean problemas en la escuela.

La perspectiva de la enseñanza centrada en la resolución de problemas asume que las interacciones entre los sujetos son un elemento fundamental en la apropiación del conocimiento matemático; entre otras razones, porque desde el origen mismo de las matemáticas se identifica el plantear y resolver problemas prácticos como eje fundamental por el cual se crearon. 
No obstante, a partir del periodo Griego la Historia nos muestra la necesidad de hacer un paso hacia delante, la evolución histórica de la matemática sitúa los métodos de razonamiento como centro de la investigación en la matemática... y la resolución de problemas como el medio más eficaz para coronar este objetivo (D’ Ambrosio, 1999: 29).

Acevedo y FalK (1996) comparten la idea de D'Ambrosio y agregan que la actividad centralque permite la construcción personal del saber matemático, desde los primeros años de la escuela hasta su culminación, es la resolución de problemas. Aseguran que un problema bien formulado o una secuencia de problemas bien diseñados permiten la profundización, extensión, exploración o investigación en el proceso de apropiación de un concepto matemático por parte del estudiante. Cada estudiante debe tener la oportunidad de formular sus propios problemas, tanto de situaciones cotidianas como matemáticas, como refuerzo a la construcción del significado de cada concepto y la adquisición de confianza para encontrar utilidad a la matemática. Lo anterior supone que los problemas deben ser contextualizados y vinculados con diferentes disciplinas del conocimiento.

En su realidad cotidiana el niño está expuesto permanentemente a una serie de eventos y situaciones problema que exigen un conocimiento en relación a las matemáticas, por ello su aprendizaje se inicia mucho antes del ingreso del niño a la escuela. Es necesario distinguir entrela lógica del contenido (matemáticas como ciencia) y los procesos de construcción del aprendizajede los niños (Guerrero, 2003).

Miranda, Navarro y Sugey (2007), y Martínez González (2007) analizan la resolución de problemas desde la óptica de las creencias y emociones que las matemáticas despiertan en los estudiantes, aseguran que el éxito de su aprendizaje radica en el gusto y el nivel de autoconfianza, es decir, el enfrentarse a una problema matemático despierta ciertas emociones de desconfianza e inseguridad que si no son controladas pueden causar bloqueos cognitivos y consecuentemente abandonar la resolución del problema.

Rubio, López y Andino (2007) plantean que en la resolución de problemas no basta la aplicación cuidadosa de reglas matemáticas. Se necesita educar la capacidad de análisis, síntesis, correlación, e intuición. López y Mochón (2007) agregan que se parte de un problema y de éste los estudiantes generan nuevos, lo que involucra el pensamiento reflexivo.

\section{DISEÑO Y METODOLOGÍA}

Recuperando el hecho de que en la investigación cualitativa coexisten diferentes formas de hacerla y con el fin de lograr el objetivo de esta investigación, que es identificar las características 
de los problemas y los ejercicios, se requiere usar el enfoque etnográfico como dispositivo metodológico, entendido como:

Una descripción o reconstrucción analítica de escenarios y grupos culturales intactos [...]recrea para el lector las creencias compartidas, prácticas, artefactos, conocimiento populary comportamiento de un grupo de personas [...] el etnógrafo comienza examinando grupos y procesos, incluso muy comunes, como si fueran excepcionales o únicos, ello le

permite apreciar los aspectos, tanto generales como de detalle, necesarios para dar credibilidad a su descripción [...] en cuanto a procesos la etnografía es uno de los modelos generales de investigación [...] para el estudio del comportamiento humano (Gotees y Le Comte, 1998: 28 - 29).

Para Bertely (2002) las investigaciones etnográficas pueden organizarse de distintas maneras gracias al contexto en que los investigadores elaboran sus particulares organizaciones, y a los problemas teóricos metodológicos o epistemológicos que les interesa resolver.

En este sentido, los estudios cualitativos manejan las percepciones subjetivas de los involucradosy el contexto sociocultural en el que se producen, así, la etnografía es una forma naturista de la investigación; cuyo objeto es combinar el punto de vista del observador interno con el externo para describir el marco social, "la etnografía es un enfoque cualitativo construido en sus orígenes por supuestos teóricos funcionalistas de la antropología y de la fenomenología en el ámbito de la sociologíacualitativa. Su articulación metodológica e instrumental se deriva de su coherencia entre estos diferentes niveles" (Reynaga, 2003: 131), sería erróneo reducirla a una mera técnica de investigación.

El proceso de investigación etnográfica implica: Acceder, mantener y desarrollar una relación con las personas generadoras de datos. Esta fase es la que generalmente se denomina acceso al campo. Exige ciertas habilidades y recursos. Emplear variedad de técnicas para reconocer el mayor número de datos y/o informaciones, aspecto que redundará en la validez y fiabilidad del estudio. Permanecer en elcampo el tiempo suficiente para asegurar una interpretación correcta de los sucesos observados y recriminar entre lo regular e irregular. Utilizar teorías y conocimientos para guiar e informar las propiasobservaciones de lo visto y oído, desarrollar hipótesis específicas y categorías de observación, definir el tema y depurar el proceso de estudio (Wilcox citado por Colás

1998: 259).

En apego al carácter flexible, característico de la etnografía, se definió el proceso metodológico a seguir en la presente investigación; cuyos pasos fueron: El objeto de estudio. Las preguntas de investigación y su análisis. Los espacios de investigación. Métodos e instrumentos de investigación. El tratamiento de la información. Primeras inferencias y conjeturas. La construccióndel informe de investigación 


\section{RESULTADOS}

Entender al problema como un obstáculo para el sujeto y que éste, no cuenta con un dispositivo inmediato para resolverlo, pero si le exige ciertas situaciones de análisis y reflexión en la definición del proceso de solución, nos conduce a diferencia un verdadero problema de situaciones similares como pueden ser los ejercicios, veamos como el docente recurrentemente utiliza el ejercicio como sinónimo de problema.

En el pizarrón se observaba. Multiplicación con números decimales.

Una familia gasta \$ 47.39 diarios ¿Cuánto gastará en 365 dias? (El alumno inmediatamente plantea el algoritmo de la multiplicación).

Escribe:

47. 39

$\underline{X 365} 123704$

28434

$\underline{14217}$

17297.44

La leyenda escrita en el pizarrón (multiplicación con números decimales) sugiere la resolución inmediata del cuestionamiento que planeta el profesor sin que el alumno invierta recursos cognitivos para definir el proceso, lo cual, reduce la situación a un ejercicio.

Sin embargo, la deficiencia está en el manejo de la técnica, entendida como el uso eficiente del procedimiento de la multiplicación y su forma de representación al efectuar los cálculos, lo cualobliga a diferenciar entre un problema y el uso de la técnica.

Visto así, el algoritmo de la multiplicación es el problema, sin embargo, sólo se trata de la ejercitación mecánica de una operación aritmética, es decir, el alumno muestra dominio sobre el valor posicional, pero carece de las mecanizaciones parciales que implican multiplicar cinco por nueve, cinco por tres, entre otros.

En múltiples momento de la investigación se observó que el manejo eficiente o deficiente de las técnicas matemáticas establece la diferencia entre quienes solucionaban los ejercicios de manera óptima y quienes lo hacían de manera deficiente, al mismo tiempo, el profesor hace un sinónimo entre problema y uso de la técnica. De esta manera pareciera que la finalidad de la enseñanza de las matemáticas es apropiarse de técnicas matemáticas y no del desarrollo de procesos cognitivos 
complejos como el uso de estrategias, la toma de decisiones, en otros, ejes centrales de la resolución de problemas.

Pero no se puede despreciar el "valor didáctico del ejercicio en el aprendizaje de la matemática porque posibilita consolidar habilidades instrumentales básicas" (Pozo 1998; p.18) el obstáculo es convertirlo en el único medio para aprender.

Continuando con el análisis de la clase podemos establecer otras relaciones entre ejercicio y problema.

En el salón se escuchan murmullos de cómo se tiene que resolver la operación.

(Mientras tanto 4 estudiantes se auxilian de los dedos de la mano para resolver el problema, tresse mantienen expectantes a lo que hace el alumno que está frente al pizarrón pero no hacen nada en su cuaderno. Al terminar el alumno).

M. ¿Está bien?

AT. No (En coro).

M. Haber Edwin pasa (Edwin escribe en el

pizarrón).

\section{2 \\ 415 \\ 324 \\ 47.39 \\ $\underline{X 365} 123597$ \\ 28416 \\ $\underline{14717}$ \\ 1728355}

(Cuando Edwin termina y se aleja del pizarrón).

A22. Maestro en el 97 está bien con el 35, pero el 12 no (se refería al resultado de lamultiplicación del primer digito del multiplicador.

El establecimiento de una sola tarea escolar para todos los estudiantes no garantiza que se planteen problemas o ejercicios, porque depende, entre otras cosas, del cúmulo de conocimientos y habilidades de cada uno, los cuales están íntimamente relacionados al contexto social y cultural de 
procedencia, así mismo, de los fines que tiene el estudiante al resolverlo, es decir, los tres alumnos que se mantienen expectantes no manifiestan la intención de hacerlo.

Lo anterior impone la necesidad de aplicar actividades de aprendizaje diferenciadas a los conocimientos y habilidades de los alumnos para poder entender cuando se resuelve un ejercicioo un problema.

La solución de problemas y la realización de ejercicios demandan de los profesores conocer los límites entre uno y otro, para poder definir las actividades didácticas que habrán de desarrollarseen el salón de clases.

Durante la investigación, el ejercicio no solo se manifiesta en el aprendizaje de las operaciones básicas de suma, resta, multiplicación y división, fue frecuente en el trazo de figuras geométricas y en el cálculo de perímetros y áreas.

El trazo de figuras geométricas se relaciona con la reproducción o construcción de gráficosque se hacen de los objetos geométricos.

La reproducción constituye una copia de un modelo dado; como se muestra en la figura 4.

Se realizó a partir de las instrucciones verbales del profesor.

Figura 1. Trazo de figuras geométricas.

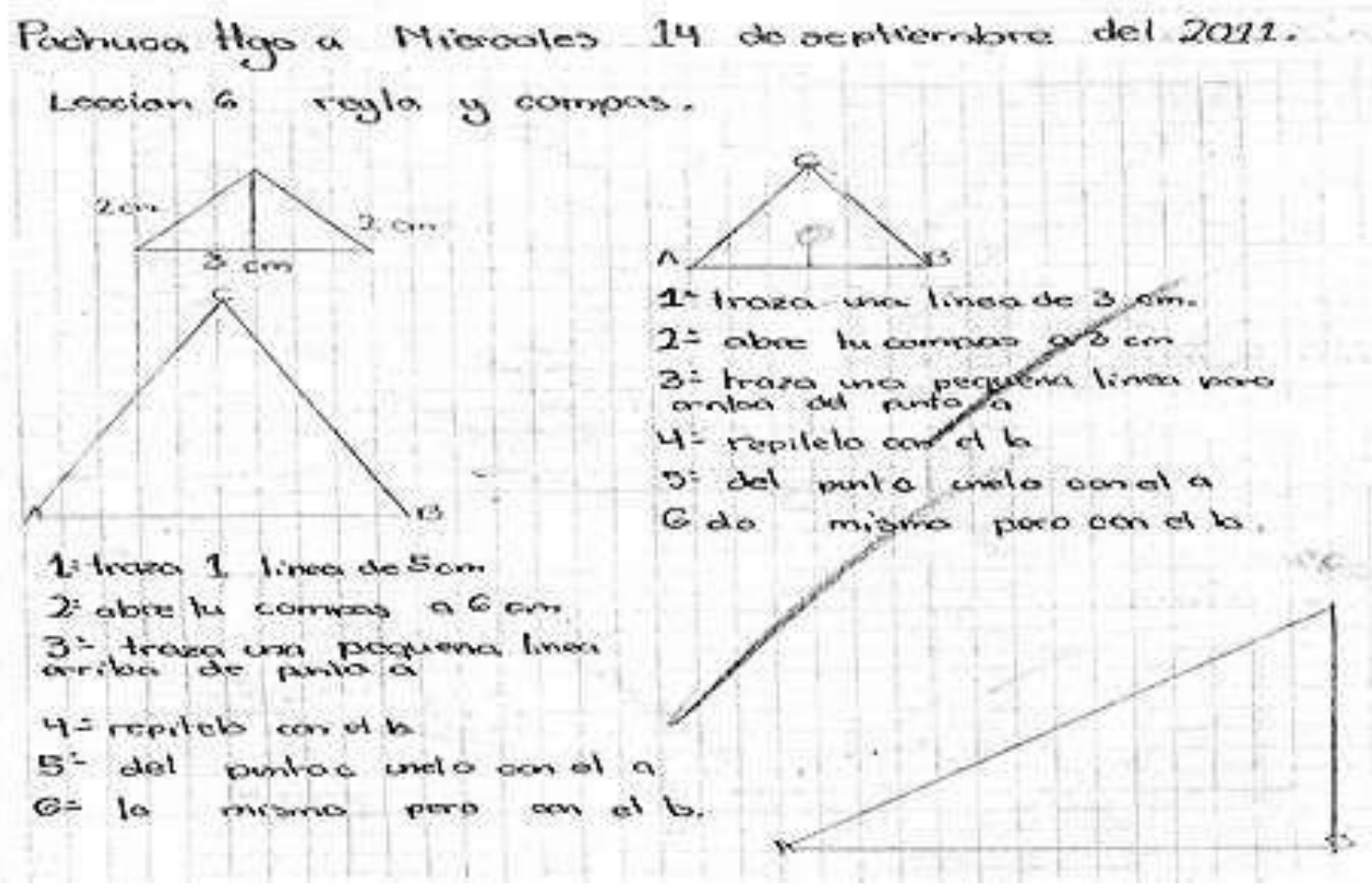

Fuente: Cuaderno del alumno.

El haber dado las indicaciones precisas lo convirtió en un ejercicio para el uso de la regla y el compás, sin embargo, limitó a los alumnos en el análisis de otras relaciones y propiedades de los 
triángulos, tales como, ángulos internos, ángulos externos, ángulos rectos, obtusos, agudo, etcétera, y con ello, seguir ejercitando y profundizando en el lenguaje matemático.

En este contexto, la figura 4 evidencia errores del profesor en el uso del lenguaje matemático; por ejemplo:

Traza una línea de 5 centímetros. La indicación debió haber sido: traza un segmento de 5 centímetros; el segmento está limitado en ambos extremos por puntos; En el trazo se definieron como A y B. Porque la línea recta tiene longitud infinita; toda línea recta que contenga puntos del interior de un círculo o de cualquier figura cerrada, necesariamente corta el borde de ese círculo o de esa figura cerrada, la línea recta es la distancia más corta entre dos puntos.

Traza una pequeña línea arriba del punto a. En realidad se tenía que trazar un arco con centro en $\mathrm{A}$ y radio $\mathrm{AB}$, un arco con centro en $\mathrm{B}$ y radio $\mathrm{AB}$, para definir el punto $\mathrm{C}$ en el lugar donde se cortan los dos arcos.

Las imprecisiones en el vocabulario matemático conducen a confusiones y deficiencias enlos procesos de comunicación matemática. Al respecto Lee (2010) seña: existen problemas que surgen del vocabulario matemático.

Las palabras utilizadas en clase de matemáticas pueden ser similares a las palabras utilizadas en la vida diaria, ocasionalmente el alumno precisa pensar en ellas de modo diferente cuandose trata de matemáticas. Esto se debe a que en matemáticas, el contexto de un problema a menudo no se pretende que sea visto como una realidad. Por ejemplo, si un problema plantea

el número de viajes que tiene que hacer un ascensor para transportar a determinada cantidad depersonas hasta la última planta, es razonable deducir que si tarda mucho, unas cuantas personas decidirán subir a pie. En un problema matemático esto no se considera cuando se intenta darcon la solución. Los problemas del "mundo real" se introducen para demostrar que esta cienciaes accesible, real y tangible. Sin embargo, la facultad de las ideas matemáticas es que son abstractas y no contextuales, y reparar en ello ayuda a los estudiantes (p. 40).

Durante la investigación se encontró que el profesor asocia el término problema al planteamiento de un enunciado en el cual se combina lenguaje materno y lenguaje matemático, y no considera el grado de complejidad como elemento fundamental de la enseñanza con problemas, en este sentido el enunciado se puede convertir en ejercicio.

Para Chamorro (2006) un enunciado matemático describe un estado o suceso, más o menos corriente, o que tiene que ver con el universo imaginario familiar del niño. A la descripción le vieneuna o varias preguntas a las que los alumnos deben responder. La situación que se describe en el enunciado está completada de una manera particular, resaltan los aspectos cuantitativos dando ciertos datos numéricos. La elaboración de la respuesta implica inferencias que ponen en marcha que ponen en 
marcha competencias de tipo lógico-matemático. La respuesta, la solución del problema, debe ser presentada de una manera particular, utilizando el lenguaje matemático.

Lo anterior hace que las dificultades de lectura del enunciado de un problema sean significativamente diferentes de las dadas en la comprensión del lenguaje común, entre otras razones, porque los enunciados matemáticos no tienen como misión clarificar el problema, esto es función del alumno con ayuda de los datos que le dan.

En la figura 5 se presenta: ¿Obtén el perímetro de un decágono regu ar que mide $16.54 \mathrm{~m}$ por lado? para solucionarlo le pide defina la formula, señalen las operaciones, presenten la sustitución y muestren el resu tado. El enunciado, para su solución, solo implica recordar el número de lados del decágono y la fórmula de su perímetro, para lo cual, sólo requiere de la mecanización esencia misma del ejercicio.

Figura 2. El ejercicio como enunciado.

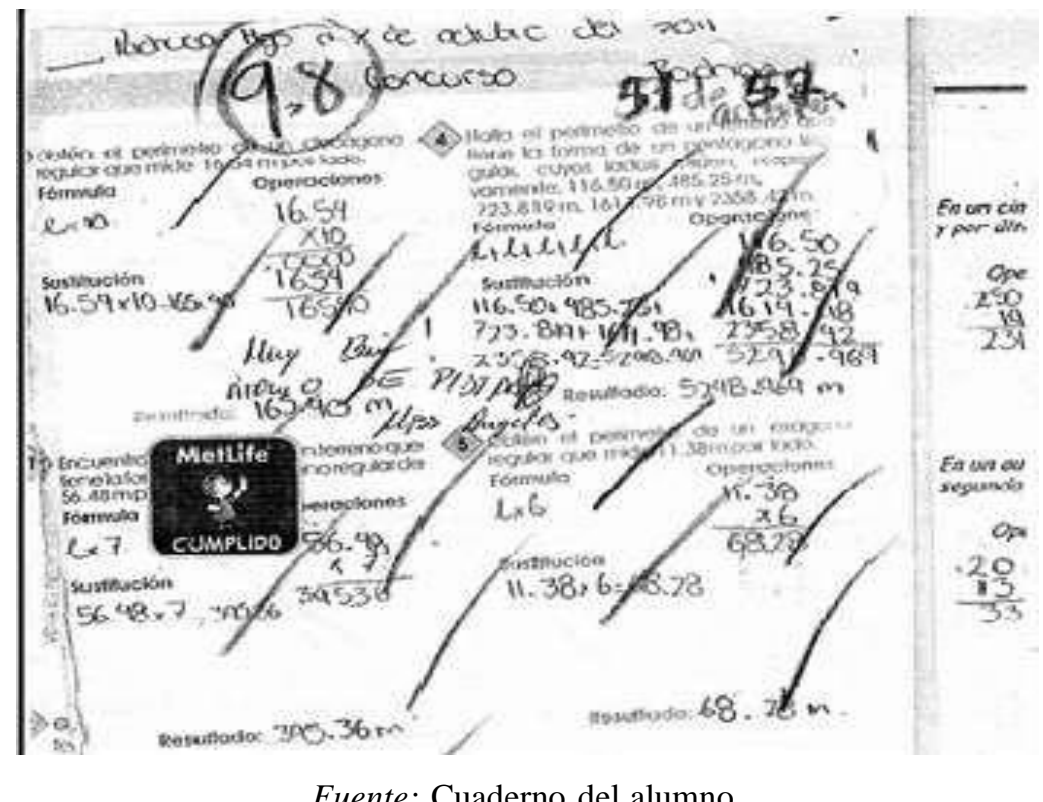

Fuente: Cuaderno del alumno.

Sin embargo, los ejercicios como enunciados, juega un papel fundamental en la construcción del lenguaje matemático, porque el alumno necesita recordar definiciones, axiomas, teoremas, propiedades, entre otros, para poder expresar sus ideas matemáticas y establecer diálogos con los demás en el discurso matemático.

$\mathrm{Al}$ respecto Lee (2010) señala:

Si los alumnos tienen que involucrarse plenamente en el discurso matemático tanto dentro como fuera del aula, deben comprender los convencionalismos del lenguaje matemático y los profesores deben ayudarles en el proceso [...] El modo en que se expresan las matemáticas en los libros de texto, al igual que por parte de muchos 
profesores no corresponde con los hábitos lingüísticos normales del alumnado. En el aula es normal oír decir a los alumnos "cuentas tantas veces el largo por el ancho y te da el área”, que la forma matemática más pasiva y convencional "el área de un rectángulo es igual a la longitud multiplicada por la anchura". Aunque ambas frases expresan el mismo concepto, la segundase considera convencionalmente más matemática, ya que se expresa en un estilo conciso, impersonal e inmutable" (p. 34).

Finalmente, las imprecisiones que surgen en la apropiación del lenguaje matemático, detienen el uso adecuado de los conocimientos por los alumnos.

\section{DISCUSIÓN}

En el currículo prescrito para quinto grado se plantean 18 contenidos donde la resolución de problemas se toma como un aprendizaje, sin embargo, siempre se asocia a un contenido específico de la matemática, por ejemplo: resolver problemas que impliquen multiplicar números fraccionarios y decimales por números enteros. Lo anterior permite al profesor, primero, ejercitar el algoritmo de la multiplicación de fracciones y decimales con números enteros y después plantear problemas para ser resueltos por los estudiantes, los cuales se convierten en mecanizaciones de nociones y procedimientos, entendida la noción como el sistema de numeración y el procedimientos como el valor posicional de la cifra, siendo diferentes en cada operación aritmética. Así la resolución de problemas pierde riqueza en el desarrollo del pensamiento matemático, al respecto Resnick y Ford (1998) y Alcalá (2002) coinciden, los ejercicios reiterados no pueden desarrollar el pensamiento cualitativo porque interpretan a la matemática como una colección de vínculos aislados, lo cual impide al sujeto singular construir un saber integrado y de principios. El ejercicio como medio para el aprendizaje sólo cultiva el adiestramiento, es decir, el aprendizaje superficial que impide el desarrollo del razonamiento y la comprensión conceptual. En tanto partir de un problema, a decir de Ausubel y Novak (2009), estimula la disponibilidad de conceptos y principios en la estructura cognitiva pertinentes para losproblemas particulares que se vayan presentando; las características cognitivas de personalidad como la agudeza, la capacidad de integración de conocimientos, flexibilidad del pensamiento, la curiosidad intelectual, la capacidad verbal y la disposición cognitiva general.

\section{CONCLUSIONES}

En este contexto, durante la investigación fue frecuente encontrar enunciados, que a decir del profesor, eran problemas, sin embargo al momento de resolverlos resultaban ser ejercicios. Locual aleja las finalidades del currículo con las prácticas de enseñanza de los profesores. El

sujeto que aprende sólo responde a los estímulos externos, el aprendizaje es la suma de relaciones o asociaciones entre estímulos y respuestas, sin ninguna organización estructural. Por ello 
n existe ningún cambio cualitativo entre un estado inferior de conocimiento con otro superior, son meras modificaciones cuantitativas.

Para Ávila (2004), la no modificación de las prácticas de enseñanza de los profesores reflejan:el nivel de conocimientos, las opciones de actualización con que han contado y por las competencias didácticas desarrolladas. De esta manera, entre lo propuesto por currículo prescritoy lo realizado en la cotidianeidad de las aulas media una serie de interpretaciones y ajustes que signan el tránsito de la utopía a la realidad de las escuelas.

\section{PROSPECTIVA}

A partir de los resultados de la investigación diseñar un proceso de intervención en aula, haciendo énfasis en la identificación, planteamiento y resolución de problemas; entendiendo al problema desde tres dimensiones: enfoque, contenido de aprendizaje y estrategia de aprendizaje. 


\section{BIBLIOGRAFÍA}

Acevedo, M., \& Falk, M. (1996). Logros para la matemática escolar: Escuela primaria (Santafé DeBogotá). Educación y Cultura, 36-37, (Marzo).

Alcalá, M. (2002). La Construcción del Lenguaje Matemático. España: Graó.

Ausbel, D., Novak, J. (2009). Psicología Educativa; Un Punto de Vista Cognitivo. Reimpresión. México:Trillas.

Ávila, A. (2003). Introducción. Saberes Científicos, Humanísticos y Tecnológicos: Procesos de Enseñanza y Aprendizaje. La Investigación Educativa en México, 1992 - 2002. Ángel D. López YMota (Coord.). México: COMIE.

Ávila, A. (2004). Introducción. La Reforma Realizada; La Resolución de Problemas como Vía delAprendizaje en Nuestras Escuelas. México: Secretaria De Educación Pública.

Ávila, A., Block, D., \& Carvajal, A. (2003). Investigaciones sobre educación preescolar y primaria. Saberes Científicos, Humanísticos y Tecnológicos: Procesos De Enseñanza y Aprendizaje. LaInvestigación Educativa en México, 1992 - 2002. Ángel D. López y Mota (Coord.). México: COMIE.

Beterly B. M. (2002). Conociendo Nuestras Escuelas; Un Acercamiento Etnográfico a la Cultura Escolar. México: Paidós.

Chamorro María Del Carmen. (2006). Didáctica de las Matemáticas. España: Pearson.

Colás, Bravo, Pilar. (1998). Enfoque en la metodología cualitativa: sus prácticas, de investigación. Métodos de Investigación en Psicopedagogía. Buendía, Leonor, \& Fuensanta Hernández (Coord.).México: Mc Graw.

D’Ambrosio, J. (1998). El problema de plantear el problema (Sevilla). Investigar en la Escuela, 35.

D’Ambrosio, J. (2005). Sociedade, Cultura, Matematica E Seu Ensino (Brasil). Educacao E Pesquisa. $31(1)$.

D’Ambrosio, J. (1999). Educacio Matematica Per A Una Civilitzacio En Proces De Calvi (España). TempsD’ Eduacio. 22 (Jul - Des).

Gotees, J. P. \& Le Compte, M. D. (1998). El Rol Del Etnógrafo. Etnografía Y Diseño Cualitativo En Investigación Educativa. Madrid: Morata.

Guerrero, A. (2007). Análisis De Las Estrategias De Solución Para Problemas Aditivos (España). Iberoamericana De Educación. (33).

Lee C. (2010). El Lenguaje En El Aprendizaje De Las Matemáticas. España: Morata.

López, I., \& Mochón, S. (2007). El Uso De Computadora Y Cañón Para El Desarrollo Del EntendimientoMatemático De Fracciones En $4^{\circ}$ De Primaria (México). Acta Latinoamericana . 
Miranda, N., Navarro, C., \& Elika S. (2007). Conflictos Cognitivos Que Emergen En La Resolución DeProblemas Relativos Al Límite (México). Acta Latinoamericana De Matemática Educativa (Clame). XX, 3 - 9.

Pozo, J. I. (1998). La Solución De Problemas. (pp. 14-50). México: Santillana.

Reynaga, S. (2003). Perspectivas Cualitativas De La Investigación En El Ámbito Educativo. La Etnografía Y La Historia De Vida. Tras Las Vetas De La Investigación Cualitativa, Perspectivas Y Acercamientos Desde La Práctica. Rebeca Mejía Y Sergio Antonio Sandoval (Coord.). México.

Resnick L., \& Ford, W. (1998). La Enseñanza De Las Matemáticas Y Sus Fundamentos Psicológicos. España: Paidós.

SEP. (1993). Libro Para el Maestro. México.

SEP. (1993). Plan y Programas de Estudio para la Educación Primaria. México.

SEP. (1999). La Enseñanza de las Matemáticas en la Escuela Primaria. Taller para Maestros. SegundaParte (Programa Nacional de Actualización Permanente). México.

SEP. (2009). Programa de Estudio para la Educación Primaria. México.

Vila, A., \& CALLEJO, Callejo, M. L. (2004). Matemáticas para Aprender a Pensar; el Papel de las Creencias en la Resolución de Problemas. México: Narcea. 